\title{
EDUCAR PARA LA PAZ CON LOS MEDIOS AUDIOVISUALES
}

\author{
Inmaculada Contreras Sedes \\ Licenciada en Bellas Artes \\ Poeta y profesora de Educación Plástica y Visual \\ Instituto de Educación Secundaria de Almagro, Ciudad Real, España \\ inmacontreras2002@yahoo.es
}

Educar para la paz parece una falacia inocentona en nuestros días. Si educar en sí mismo es complejo, introducir en el currículo escolar la idea de la educación para la paz, puede sonarnos hasta pretencioso. No nos engañemos, educar para la paz es cualquier cosa menos una tarea sencilla.

Sin embargo, introducir en la educación las ideas de la Conferencia General de la UNESCO, en su Declaración de Ia XLIV reunión de la Conferencia Internacional de Educación, de tratar de introducir en el éxodo educativo los resortes necesarios para que se mengüen las brechas entre ricos y pobres, las manifestaciones de violencia, racismo, xenofobia, o las violaciones de los derechos humanos, o la intolerancia de cualquier índole, las más de las veces no es sino un montón de buenos deseos, o, en el mejor de los casos, una excusa en los colegios o en los centros de Secundaria para celebrar el Día de la paz y la no violencia, un día que se llena de dibujitos de palomas y fichas para dibujar arcoíris (...) y poco o algo más.

¿Es eso educar para la pazo una excusa para decorar los centros con frases estereotipadas y manidas? ¿No es un modo de escribir cuatro pamplinas de tema edulcorado, $y$, eso sí, bellamente decoradas? Muchas veces caemos en este tipo de "reflexiones".

Desde mi punto de vista, educar para la paz debe implicar algo más profundo: debe atender a la idea, no sólo de prevenir el conflicto, sino también de conocer el sabor mismo del conflicto, para ser conscientes de que lo más sensato y humano es, precisamente, evitarlo.
Si, como afirma Galtung (1980), "La paz es un camino que se define como la capacidad de manejar los conflictos con empatía, no violencia y creatividad" nuestro modo de enseñar no puede sino tratar de desarrollar en nuestro estudiantado la capacidad de ponerse en la piel del otro, independientemente de la cercanía o lejanía de sus realidades, la necesidad de vivir en paz, no como una utopía, sino como una meta que es necesario conseguir, y la creatividad para que este aprendizaje resulte divertido, y cale como la lluvia, poquito a poco, y de ese modo logremos que el estudiante fagocite estas ideas, las digiera, y las incorpore a su propio ADN.

¿Cómo lograr estos objetivos? Desde la Eskola Bakegune, nos proponen educar en y para el conflicto, desde una triple perspectiva:

- Descubrir la perspectiva positiva del conflicto, verlo como una forma de transformar la sociedad y las relaciones humanas hacia mayores cotas de justicia y descubrirlos como una oportunidad educativa, una oportunidad para aprender a construir otro tipo de relaciones, así como para prepararnos para la vida y aprender a hacer valer y respetar nuestros derechos de una manera no violenta.

- Aprender a analizar los conflictos y a descubrir su complejidad, dando pautas al profesorado, padres/madres y estudiantado para que tengan herramientas que les ayuden a enfrentar y resolver los conflictos en los que nos vemos inmersos cotidianamente. 

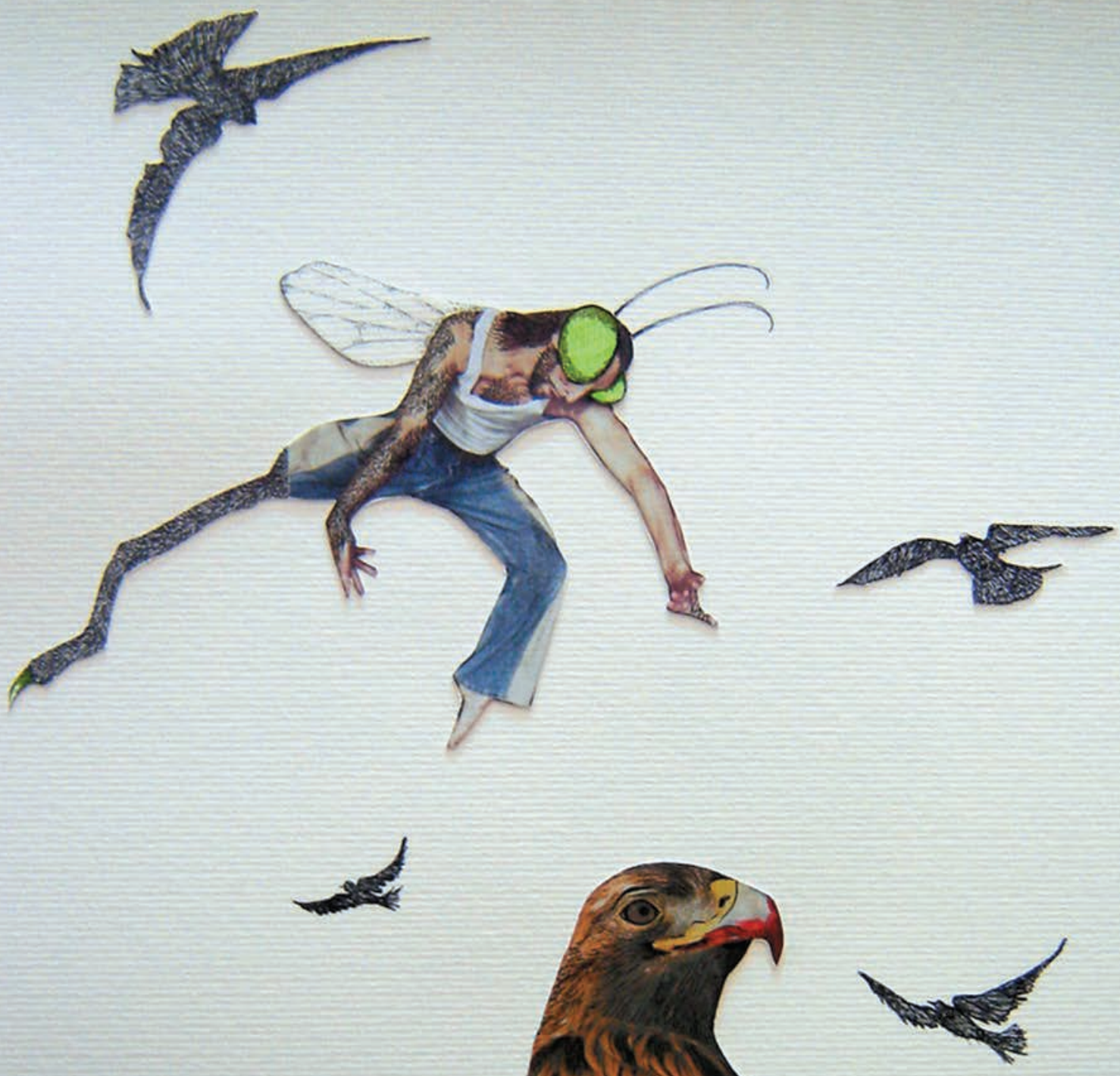
- Encontrar soluciones que nos permitan enfrentar los conflictos sin violencia, sin destruir a una de las partes y con la fuerza necesaria para llegar a soluciones en las que todos y todas ganemos, favoreciendo por sobre todas las cosas la asertividad.

Y dicho todo esto, tenemos un marco envidiable para trabajar la paz en el entorno escolar. Realmente lindo, pero: ¿De qué modo llevamos estas bonitas teorías a la realidad el aula? En mi caso el uso de los medios audiovisuales ha sido el camino que nos ha llevado a acercarnos de modo práctico y lúdico a trabajar estas temáticas. Os cuento mi experiencia, por si os sirve.

Desde el reducido horario que me permiten mis clases, dos horas a la semana, y usado a intervalos también el tiempo dedicado a la acción tutorial, he trabajado la educación emocional transversalmente, imbricada en la enseñanza de mi área, la Educación Plástica y visual, a través del trabajo cooperativo, asumida la heterogeneidad del estudiantado y su diversidad, a veces incluso cultural, como caldo de cultivo para la realización de estas actividades.

En un primer momento, la tarea es muy fácil: visionar cortos cinematográficos, o una película completa en varias sesiones, previamente seleccionadas por la profesora, que incidan de algún modo en un aspecto del que sea preciso, en este caso, eran filmes que se caracterizaban por tener como trasfondo conflictos o guerras, o en las que la violencia en la sociedad era muy patente.

A partir de ahí desarrollamos un análisis verbal, con una tormenta de ideas, acerca de qué nos ha parecido, qué nos sugiere, cómo nos sentimos si nos ponemos en la piel de tal o cual personaje, (...) el debate debe estar dirigido suficientemente, pero debe dar lugar a que el estudiantado se exprese, ese es el objetivo. Un primer acercamiento al pensamiento crítico. Aquí empiezan las dificultades.

Posteriormente, con métodos de trabajo colaborativo, se trabaja en pequeños grupos

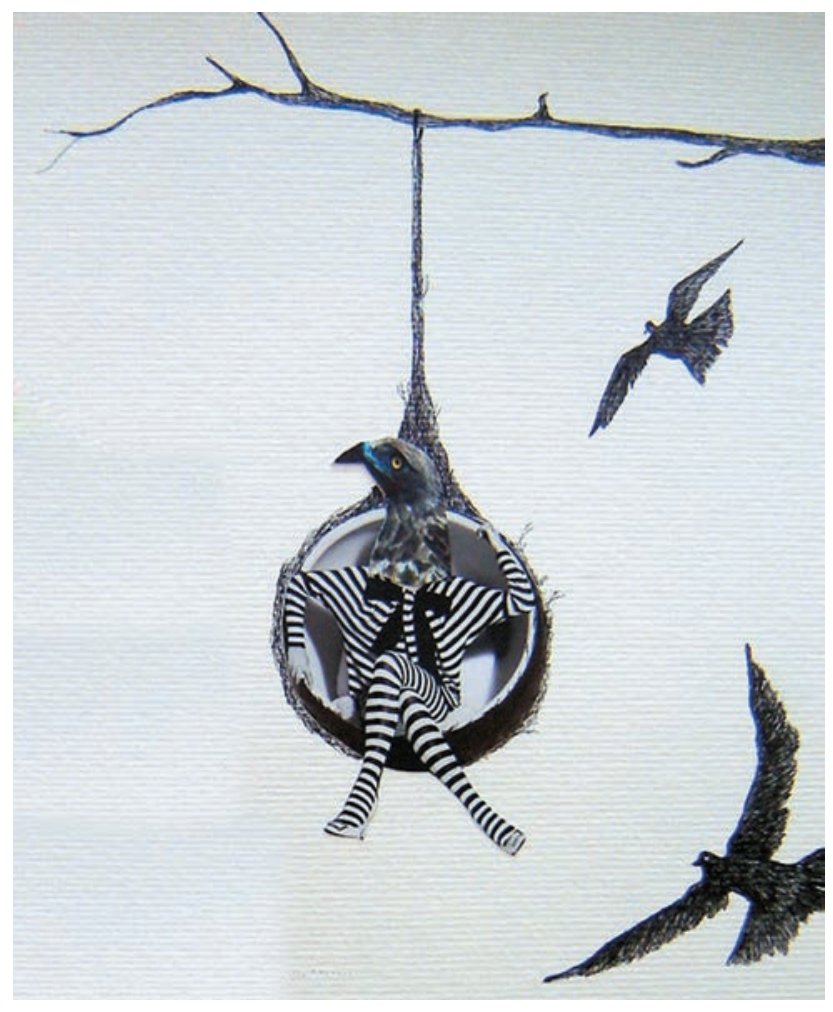

y con los medios audiovisuales, habitualmente sencillos audios o videos que realizan ellos con las cámaras de sus teléfonos celulares, los estudiantes recogen en unos casos entrevistas a amigos, familiares, vecinos, y/o gente cerca$\mathrm{na}$, en las que recopilan grabaciones en las que ellos interrogan acerca de sus experiencias en la vida diaria sobre las relaciones entre iguales, o crean sus propias versiones de determinadas escenas del film, o se inventan historias relacionadas más o menos directamente con la temática de la película visionada. No usamos siempre el mismo método, ni vale por igual para todos los grupos de alumnos, pero como regla general nos sirve para reflexionar a posteriori sobre las relaciones desiguales, la justicia de que estas existan, el maltrato verbal o físico, el acoso, o las posibles agresiones de cualquier tipo, entre otros.

El realizar grabaciones de sus propias experiencias al respecto o las de gente cercana, les ayuda grandemente a detenerse en la realidad que se les está mostrando de forma diferente: desde detrás de una cámara o un micrófono. Esto les lleva a tomarse su tiempo, a pensar, 
que al fin y al cabo es nuestro objetivo como docentes, tratar de hacer pensar a nuestros discípulos, enseñarles a que se hagan preguntas, marcar las pautas, el sendero, intentar, aportar nuestro granito de arena para que no sean parte de una masa borreguil y adocenada que se deja llevar, sino que sean conscientes y que esa conciencia les abra los ojos a lo imprescindible del respeto a los derechos humanos y a asimilar lo necesario de buscar la paz en su vida diaria.

Y ese demorarnos en el pensamiento ayuda tanto al docente, como al discípulo a crear y recrear la nueva realidad en la que, cuando menos, pensarás en el otro antes de proseguir con la habitual cadena de abusos que, las más de las veces, caracteriza a la sociedad que vivimos.

\section{BIBLIOGRAFÍA}

Galtung, J (1980) The Basic Needs Approach, en Katrin Lederer, David Antal y

Galtung, J (Eds), Human Needs: A Contribution to the Current Debate, Cambridge

(Massachusetts), Oelgeschlager, Gunn \& Hain; Koningstein, Anton Hain.

\section{Webgrafía}

Asamblea general de las Naciones Unidas: http://www. un.org/es/ga/62/plenary/sport/bkg.shtml

Declaración de la 44a reunión de la Conferencia Internacional de Educación

http://www.unesco.org/cpp/sp/declaraciones/educacion.htm

Eskola Bakegune http://www.eskolabakegune.euskadi. net 